tions have been introduced. In these the attack is still directed at the thalamo-frontal projections, but is limited in its extent. Thus in bimedial leucotomy the incision is confined to the medial halves of both frontal lobes in the plane described above. In cortical undercutting the cortex on the orbital, supero-lateral, or medial surfaces of the frontal lobes is separated from the underlying white matter. In topectomy areas of frontal cortex are excised (in particular, areas 9 and 10 of Brodman). These procedures are to be considered as allowing of a quantitative reduction in the extent of the leucotomy, there being little evidence as yet that any of the modifications is specific in the treatment of psychoses, neuroses, or intractable pain.

\section{Extra Vitamins and Colds}

Q.-Claims are made that the issue of halibut-liver oil capsules to industrial workers is beneficial in that they prevent upper respiratory tract infections. Is there any evidence that on present-day standards of nutrition this is worth doing?

A.- There is no reason to believe that the addition of extra vitamin $D$ to a diet which contains an adequate amount of this vitamin will prevent upper respiratory tract infections.

\section{Treatment of Recurrent Boils}

Q.-What is the best treatment for recurrent boils? Have vaccines any place, and, if so, are autogenous any better than stock vaccines?

A.- The treatment of recurrent boils is the treatment of the cause. The first consideration is the general health, and especially the exclusion of any organic disease such as diabetes or anaemia or any cause of lowered resistance. The second is the exclusion of any source of infection in the patient. This may be a septic focus such as a tooth, or a skin lesion such as flexural eczema or pruritus ani. It is easy to overlook the presence of parasites, since they are uncommon to-day. Thirdly, the environment should come under review. The most important feature here is possible contact with an individual suffering from boils or other sepsis, or with something which such a person handles, including food.

When there is furunculosis pyogenic organisms multiply over the skin of the body, increasing the liability to boils, so that personal hygiene is important ; a daily bath followed by a zinc oxide dusting powder and a daily change of underlinen, which is boiled, should be enjoined. If localized, $x$-ray therapy or dry heat and a non-irritating antiseptic lotion such as $1 \%$ cetrimide may help. Empirically, the administration of iron and of vitamin B is recommended, cocoa fat and pig fat being avoided. The writer has not used vaccines; those who employ them use autogenous vaccines.

\section{Lead Douches}

Q.--Solutions of lead subacetate have been recommended for douching. What are the chances of the lead being absorbed through the vaginal mucous membrane?

A. - The vaginal "mucous membrane" is a stratified epithelium similar to that of the skin except that it has no glands and that its keratin layer is thin or absent. Lead salts can be absorbed through the body surface, but not to a significant extent unless they are regularly rubbed into the skin by way of ointments or lotions. Under the latter conditions poisoning has been described, but it is exceptional Absorption through the vaginal epithelium must be accepted as a possibility, but it is a very remote one when the application is fleeting and superficial, as it would be in the case of douching, and provided the vaginal skin is unbroken. Reference books mention that cases of lead poisoning following prolonged vaginal douching have occurred, but they give no details. One of the difficulties would be to be sure that such poisoning was the result of vaginal absorption rather than the result of transfer of lead to the alimentary or respiratory tracts from the patient's hands, contaminated during the preparation and use of the douche. Provided the usual hygienic precautions are taken to exclude such transfer, douching with lead subacetate would appear to carry a negligible risk.

\section{Dangers of Paraffin Mist}

Q.-A patient of mine uses an apparatus, known as a high-pressure paraffin washer, which squirts a jet of finely divided paraffin on engine parts, etc., for cleaning. Is the inhalation of the finely divided paraffin likely to be harmful? What is the best protection for him?

A.- No general survey of the health of Workers who clean engine parts and castings by spraying them with paraffin under high pressure has yet been carried out. But there is a good deal of evidence that when mineral oils are introduced into the lungs they can set up lipoid or oil pneumonia, and in some cases a generalized fibrosis may develop. The literature on lipoid pneumonia following the use of nasal drops containing mineral oils is extensive, and there has been in this country at least one case (unpublished) in which widespread pulmonary fibrosis was found at necropsy in a man who for about 20 years had been spraying castings with paraffin. Incidentally, when paraffin is ingested, pneumonia may also occur. ${ }^{1}$ Some paraffins might contain carcinogenic substances and there is therefore a potential risk of carcinoma of the bronchus or alveoli. In some workers using the mineral-oil spray-gun there have been complaints of dizziness and loss of appetite, and others have contracted dermatitis. In high concentrations the vapours of some paraffins exert a narcotic action on the central nervous system. When the exposure is severe the symptoms may vary from dizziness and unconsciousness to convulsions, but such cases are rare.

The best protective measure would be not to use the spray method at all, because all sprays are notoriously difficult to control. But, unless the work can be done in a booth under efficient exhaust ventilation, the wearing of a respirator fitted with a fresh-air line would prevent inhalation of the atomized oil. The wearing of dust respirators such as the Mark IV or the Microfilter would afford some measure of protection. In addition, protective clothing should be worn and the skin carefully washed daily or at more frequent intervals.

$$
1 \text { Gelfand, M. (1949). British Medical Journal, 2, } 1151 .
$$

Correction.-The figures on the abscissa of the graph (Fig. 4) accompanying the article "Maximum Urine Concentration" by Drs. B. E. Miles, A. Paton, and H. E. de Wardener (Journal, October 16, p. 901) represent the solute output rate in milliosmols $/ \mathrm{min}$., not osmols $/ \mathrm{min}$.

Refresher Course Books. - The first two volumes of collected articles from the Refresher Course for General Practitioners published in the Journal are now available. The first volume contains 55 articles and the second 60 . Each article has been revised by its author. Copies of both volumes may be obtained, price 25s. (postage 1s.) each, direct from the Publishing Manager, B.M.A. House, Tavistock Square, London, W.C.1, or from booksellers. The second book of "Any Questions?" is also available, price 7s. 6d. (postage 6d.).

All communications with regard to editorial business should be addressed to THE EDITOR, British MEdical Journal, B.M.A. House. Tavistock SQUARE, LONDON, W.C.1. TELEPHONE: EUSTON 4499. TELEGRAMS Aitiology, Westcent, London. ORIGINAL ARTICLES AND LETTERS forwarded for publication are understood to be offered to the British Medical Journal alone unless the contrary be stated.

Authors desir:ng REPRINTS should communicate with the Publishing Manager, B.M.A. House, Tavistock Square, W.C.1, on receipt of proofs Authors overseas should indicate on MSS, if reprints are required, a proofs are not sent abroad.

ADVERTISEMENTS should be addressed to the Advertisement Manager B.M.A. House, Tavistock Square. London, W.C.1 (hours 9 a.m. to B.M.A. House, Tavistock Square, London, W.C.1 (hours 9 a.m. to
5 p.m.). TELEPHONE: EUSTON 4499. Westcent, London.

MEMBERS' SUBSCRIPTIONS should be sent to the SECRETARY of the Association. TELEPHONE: EUSTON 4499. TELEgRAMS: Medisecra. Westcent, London.

B.M.A. Scotrish OfFICE: 7, Drumsheugh Gardens, Edinburgh 